\title{
Prevalência de cárie dentária em dois municípios do Rio Grande do Sul, Brasil, no ano de 2010
}

\section{Prevalence of dental caries in two municipalities of Rio Grande do Sul, Brazil, in year of 2010}

\author{
Denise Paiva da Rosa* \\ Ana Paula da Silva** \\ Eduardo Dickie de Castilhos ${ }^{* * *}$ \\ Tania Izabel Bighetti***
}

\section{Resumo}

Objetivo: esta pesquisa teve como objetivo conhecer a prevalência da cárie dentária e as necessidades de tratamento em crianças de cinco e 12 anos de idade nos municípios de Sobradinho e Tavares, RS, no ano de 2010. Materiais e método: para tanto, realizou-se um estudo transversal descritivo, utilizando-se recomendações da Organização Mundial da Saúde (OMS), com dois examinadores previamente calibrados (Kappa de 0,92 interexaminadores; 0,97 para examinador A e 0,92 para examinador B). Foram calculados os índices ceod e CPOD, além do SiC Index para as duas idades e o CPOD para os primeiros molares permanentes. Resultados: foram examinadas 115 crianças em Sobradinho e 91 em Tavares. Estavam livres de cárie aos 5 anos $32,4 \%$ das crianças de Sobradinho e $45,7 \%$ das crianças de Tavares. O CPOD foi de 1,05 em Sobradinho e 2,80 em Tavares. O SiC Index para 5 anos foi de 7,23 em Sobradinho e 8,30 em Tavares enquanto que, para 12 anos, 3,27 e 5,64, respectivamente. A necessidade de tratamento predominante nos dois municípios, tanto para 5 anos quanto para 12 anos foi a remineralização. Conclusão: os dois municípios não atingiram as metas da OMS para o ano de 2010 em crianças de 5 anos. No que diz respeito às de 12 anos, o município de Sobradinho está bem próximo de alcançar o índice desejado. As necessidades de tratamento foram de baixa complexidade para os dois municípios. Verificou-se a necessidade de diagnóstico precoce, bem como de ações preventivas coletivas e individuais, voltadas para pré-escolares, uma vez que os primeiros molares permanentes foram responsáveis por uma considerável porcentagem da composição do índice CPOD aos 12 anos.

Palavras-chave: Levantamentos epidemiológicos. Cárie dentária. Saúde da criança.

\section{Introdução}

A cárie dentária, apesar de seu acentuado declínio em crianças e adolescentes, observado tanto mundial quanto nacionalmente, continua sendo o principal problema de saúde bucal a ser enfrentado no Brasil ${ }^{1}$. Dessa forma se torna importante conhecer os níveis em que a cárie dentária afeta a população, bem como as necessidades de tratamento existentes.

Para o planejamento de serviços de saúde, é importante o uso de dados e informações epidemiológicas, as quais podem fornecer o conhecimento acerca da prevalência e da tipologia das doenças bucais, possibilitando, a partir dos dados coletados, o planejamento, a executação e a avaliação das ações de

\footnotetext{
Cirurgiã-dentista graduada pela Faculdade de Odontologia da Universidade Federal de Pelotas, Pelotas-RS, Brasil.

Cirurgiã-dentista graduada pela Faculdade de Odontologia da Universidade Federal de Pelotas, Pelotas-RS, Brasil.

Doutor em Epidemiologia, Departamento de Odontologia Social e Preventiva, Faculdade de Odontologia da Universidade Federal de Pelotas, Pelotas-RS, Brasil.

**** Doutora em Saúde Pública, Departamento de Odontologia Social e Preventiva, Faculdade de Odontologia da Universidade Federal de Pelotas, Pelotas-RS, Brasil.
} 
saúde $^{2}$, permitindo que essas sejam adequadas à realidade local ${ }^{3}$.

A Organização Mundial da Saúde (OMS) recomendou que estudos epidemiológicos deveriam ser realizados a cada cinco anos ${ }^{4,5}$, dando uma noção da importância de se buscar a recuperação da saúde da população, usando métodos preventivos ou curativos. Destaca, também, que as ações devem ser baseadas em dados locais, os quais permitem a identificação dos problemas mais prevalentes naquela comunidade, a fim de se obter um adequado acompanhamento das condições de saúde bucal, bem como a avaliação das ações desenvolvidas. A OMS ainda sugeriu algumas idades-índices para serem usadas nos levantamentos, entre elas as de 5 e 12 anos.

Em 2003, foi realizado, no Brasil, um estudo de abrangência nacional com o objetivo de conhecer a predominância de algumas doenças bucais, dentre elas, a cárie dentária. Os resultados obtidos no estudo revelaram um índice de CPOD médio aos 12 anos de idade de 2,78, sendo o componente relativo aos "dentes cariados" o mais significativo (60,8\%), e com $70,7 \%$ das necessidades de tratamento representadas por restaurações de uma ou duas superfícies. Para 5 anos de idade, o índice ceod médio foi de 2,80 , tendo o componente referente aos "dentes cariados" também como o mais significativo, representando mais de $80 \%$, e com $79,7 \%$ das necessidades de tratamento representadas por restauração de uma ou duas superfícies ${ }^{6}$.

Segundo dados da Pesquisa Nacional em Saúde Bucal do Brasil (SB Brasil 2010), o CPOD para 12 anos de idade foi de 2,1 e, para a idade de 5 anos, o ceod foi de 2,3, o que mostrou um declínio de $26 \%$ e $17 \%$ respectivamente, quando comparado ao SB Brasil 2003 $3^{1,6}$. Nos estudos multicêntricos realizados no Brasil ${ }^{1,6}$, os municípios de Sobradinho Tavares não foram incluídos nas amostras de suas macrorregiões, pois a participação no SB Brasil foi definida através de sorteio.

Não há relatos de levantamentos epidemiológicos referentes às condições de saúde bucal dos dois municípios, os quais possam auxiliar no direcionamento da atenção aos possíveis grupos mais acometidos e uma posterior comparação com levantamentos futuros. Por conta dessa constatação, este estudo teve como objetivo conhecer a prevalência da cárie dentária e as necessidades de tratamento, decorrentes deste agravo em crianças de 5 e 12 anos de idade nos municípios de Sobradinho e de Tavares.

\section{Materiais e método}

Foi realizado um estudo observacional transversal, de caráter descritivo, com a coleta de dados primários, no período de agosto a setembro de 2010 nos municípios de Sobradinho (14.283 habitantes) e Tavares (5.351 habitantes), ambos no Rio Grande do Sul. Essa pesquisa fez parte de outro estudo, mais amplo, que também verificou a prevalência de fluorose dentária e identificou de forma exploratória as atuais exposições a produtos fluorados.

$\mathrm{O}$ modelo de assistência vigente, nesses municípios, se caracteriza por um elevado número de procedimentos curativos, um considerável número de procedimentos individuais preventivos e um número reduzido de procedimentos coletivos ${ }^{7}$, o qual é inferior ao ideal para obtenção de um modelo com ações preventivas coletivas e individuais não invasivas, assim como para o controle da doença e para o desenvolvimento de ações de tecnologia invasiva para o controle da lesão ${ }^{8}$.

A população de estudo no município de Sobradinho foi constituída de uma amostra de conveniência, composta por crianças de 5 e 12 anos de idade da rede municipal de ensino, selecionadas a partir das listas das 14 escolas municipais de ensino da área urbana e rural, fornecidas pela Secretaria Municipal de Educação (SME), bem como com base na listagem de duas escolas estaduais de ensino da área urbana do município fornecidas pelas suas direções.

No município de Tavares, foi realizado um censo dos escolares de 5 e 12 anos de idade da rede municipal e estadual de ensino. A seleção, nesse caso, tomou por base as listas das cinco escolas municipais das áreas urbana e rural e de uma escola estadual, as quais foram fornecidas pela direção da escola estadual e pela SME.

Para os dois municípios, foram considerados com 5 anos de idade os nascidos em 2005 e com 12 anos de idade os nascidos no ano de 1998, conforme a data de nascimento registrada na ficha de matrícula fornecida pela instituição de ensino.

As variáveis utilizadas neste estudo foram: sexo (masculino e feminino), idade (5 e 12 anos), município, cárie dentária e necessidades de tratamento decorrentes da presença de cárie. Foram adotados códigos e critérios preconizados pela OMS para levantamentos epidemiológicos em saúde bucal ${ }^{5}$. Consideraram-se cariados apenas os dentes que apresentaram cavidades. Os dentes com mancha branca de cárie, apesar de serem considerados hígidos, podem receber apenas a indicação de tratamento remineralizador. Foram consideradas livres de cárie, as crianças que apresentaram ceod igual a zero e CPOD igual a zero.

Dois examinadores passaram por um processo de treinamento e de calibração que foi dividido em uma etapa teórica e duas etapas práticas com crianças em uma escola não participante do estudo. Os resultados da calibração foram avaliados pelo coeficiente Kappa ${ }^{9}$. O grau de concordância para cárie dentária interexaminadores foi de 0,92, intra-examinador de 0,97 para o examinador A e 0,92 para o examinador B. Os exames bucais foram realizados utilizando-se espelho plano e sonda WHO 126 (CPI) esterilizados. O local da coleta dos dados foi em salas cedidas pelas escolas, o mais próximo pos- 
sível de uma fonte de luz natural, com o examinador sentado e a pessoa examinada deitada. A limpeza e a esterilização do material foram realizadas na Faculdade de Odontologia da Universidade Federal de Pelotas (FO-UFPel) e nas unidades básicas de saúde de Sobradinho e de Tavares.

Os dados foram digitados no programa Excel versão 2007 e validados no programa Epi Data versão 3.1. Para a obtenção das frequências relativas e absolutas de cada uma das modalidades das variáveis de estudo, utilizou-se o programa Epi Data Analysis. Foram calculadas as médias dos índices ceod (cinco anos de idade) e CPOD (12 anos de idade) e seus componentes. Essas médias também foram calculadas para os primeiros molares permanentes nas duas idades. Foi avaliado para as duas idades o Significant Caries Index (SiC Index) que seleciona o terço da população mais afetado pela cárie ${ }^{10,11}$.

O projeto de pesquisa foi aprovado pelo Comitê de Ética em Pesquisa da Faculdade de Odontologia-UFPel através do parecer n. 159/2010. Foram respeitados todos os aspectos relativos a pesquisas com seres humanos previstas na Resolução 196/96 do Conselho Nacional de Saúde. O termo de consentimento continha informações sobre manutenção de sigilo, possibilidade de desistência, benefícios e não malefícios da pesquisa. Também uma autorização foi solicitada às secretarias municipais de educação, secretarias municipais de saúde dos municípios e às escolas estaduais envolvidas na pesquisa mediante uma carta de apresentação e a entrega de resumo do projeto.

\section{Resultados}

Em Sobradinho, foram examinados um total de 115 crianças de 5 e 12 anos de idade, que representaram $62,2 \%$ dos indivíduos dessas idades no município. Os 37,8\% das crianças restantes não foram examinadas, devido ao fato de não terem devolvido as autorizações ou por não estarem presentes em sala de aula no dia do exame. No município de Tavares, 91 crianças de cinco e 12 anos foram examinadas, o que representou $91,9 \%$ das crianças dessas idades. Os $8,1 \%$ perdidos se deveram ao fato de recusarem-se a participar do estudo ou por não estarem presentes no dia da coleta de dados. Os dados de caracterização da amostra, em relação ao sexo e à idade, estão descritos na Tabela 1 .
Tabela 1 - Caracterização da amostra segundo sexo e idade nos municípios de Sobradinho e de Tavares, RS, 2010

\begin{tabular}{l|r|r|r|r}
\cline { 2 - 4 } & \multicolumn{4}{c}{ Município } \\
\cline { 2 - 5 } & \multicolumn{2}{c}{ Sobradinho } & \multicolumn{2}{c}{ Tavares } \\
\hline 5 anos & & & $\mathrm{n}$ & \multicolumn{1}{c}{$\%$} \\
Sexo & 40 & 54,1 & 18 & 51,4 \\
Masculino & 34 & 45,9 & 17 & 48,6 \\
Feminino & 74 & 100,0 & 35 & 100,0 \\
Total & & & & \\
\hline 12 anos & & & & \\
Sexo & & & & \\
Masculino & 14 & 34,1 & 30 & 53,5 \\
Feminino & 41 & 100,0 & 56 & 100,0 \\
\hline Total & 115 & 100,0 & 91 & 100,0 \\
\hline Total & & & & \\
\hline
\end{tabular}

Em relação à prevalência da cárie dentária aos cinco anos de idade, a proporção de escolares livres de cárie foi respectivamente de $32,4 \%$ e de $45,7 \%$ em Sobradinho e Tavares, as médias do índice ceod foram semelhantes nos dois municípios: 2,92 em Sobradinho e 2,91 em Tavares. O componente cariado teve maior prevalência nos dois municípios, mas em Sobradinho foi o responsável pela composição quase total do índice. Em Tavares, os componentes "perdido" e "obturado" apresentaram maior percentual do que em Sobradinho. Em Sobradinho, aos cinco anos de idade, os escolares examinados tinham a totalidade de primeiros molares permanentes hígidos. Em Tavares essa proporção foi de 95,8\%. O SiC Index para os dentes decíduos foi de 7,23 e 8,30, respectivamente para Sobradinho e Tavares (Tab. 2).

Tabela 2 - Experiência de cárie (ceod, SiC Index, CPOD) em crianças de cinco anos de idade. Municípios de Sobradinho e Tavares, $R S, 2010$

\begin{tabular}{|c|c|c|c|c|}
\hline \multirow{3}{*}{$\begin{array}{l}\text { Experiência de } \\
\text { cárie }\end{array}$} & \multicolumn{4}{|c|}{ Município } \\
\hline & \multicolumn{2}{|c|}{ Sobradinho } & \multicolumn{2}{|c|}{ Tavares } \\
\hline & Média & $\mathrm{DP}$ & Média & $\mathrm{DP}$ \\
\hline hígidos (h) & 16,54 & 3,91 & 16,60 & 4,10 \\
\hline cariados (c) & 2,81 & 3,35 & 2,28 & 3,09 \\
\hline extraídos (e) & 0,03 & 0,16 & 0,31 & 0,99 \\
\hline obturados (o) & 0,08 & 0,40 & 0,37 & 0,97 \\
\hline ceod & 2,92 & 3,38 & 2,91 & 3,86 \\
\hline SiC Index & 7,23 & 3,11 & 8,30 & 2,63 \\
\hline \multicolumn{5}{|c|}{$1^{\circ}$ Molares permanentes } \\
\hline Hígidos (H) & 0,26 & 0,74 & 0,65 & 1,36 \\
\hline Cariados $(\mathrm{C})$ & - & - & 0,03 & 0,17 \\
\hline Perdidos (P) & - & - & - & - \\
\hline Obturados (O) & - & - & - & - \\
\hline CPOD & - & - & 0,03 & 0,17 \\
\hline
\end{tabular}


Aos 12 anos de idade, os percentuais referentes às crianças livres de cárie foram respectivamente de $56,1 \%$ e $16,1 \%$ em Sobradinho e Tavares. As médias do índice CPOD foram de 1,05 em Sobradinho e 2,80 em Tavares. O componente restaurado teve maior prevalência no município de Tavares, enquanto que em Sobradinho a composição do índice ficou dividida entre cariados e restaurados. O $\mathrm{SiC}$ Index foi de 3,27 e 5,64 respectivamente para Sobradinho e Tavares. Os primeiros molares permanentes representaram $82,8 \%$ da composição do índice CPOD em Tavares e 76,2\% da composição do índice CPOD em Sobradinho. Os molares hígidos representaram 77,7\% em Sobradinho e em Tavares essa proporção foi de 40,3\% e o componente restaurado dos primeiros molares representou $61,2 \%$ da composição do índice CPOD em Sobradinho e 78,4\% em Tavares (Tab. 3).

Tabela 3 - Experiência de cárie (CPOD e SiC Index) em crianças de 12 anos de idade nos municípios de Sobradinho e de Tavares, $R S, 2010$

\begin{tabular}{l|c|c|c|c}
\hline \multirow{2}{*}{$\begin{array}{c}\text { Experiência de } \\
\text { cárie }\end{array}$} & \multicolumn{5}{c}{ Município } \\
\cline { 2 - 5 } & \multicolumn{2}{|c}{ Sobradinho } & \multicolumn{2}{c}{ Tavares } \\
\cline { 2 - 5 } Média & \multicolumn{1}{c}{ DP } & Média & DP \\
\hline Hígidos (H) & 22,44 & 5,26 & 22,25 & 3,52 \\
\hline Cariados (C) & 0,46 & 1,03 & 0,64 & 1,23 \\
\hline Perdidos (P) & 0,02 & 0,16 & 0,07 & 0,26 \\
\hline Obturados (O) & 0,56 & 1,16 & 2,09 & 1,81 \\
\hline CPOD & 1,05 & 1,53 & 2,80 & 1,93 \\
\hline SiC Index & 3,27 & 1,19 & 5,64 & 0,81 \\
\hline \multicolumn{5}{|c}{ Molares permanentes } \\
\hline Cariados (C) & 2,84 & 1,06 & 1,97 & 1,43 \\
\hline Perdidos (P) & 0,29 & 0,55 & 0,45 & 0,94 \\
\hline Obturados (O) & 0,02 & 0,15 & 0,05 & 0,22 \\
\hline CPOD & 0,80 & 0,80 & 1,82 & 1,46 \\
\hline
\end{tabular}

Em relação a tratamento para as crianças de cinco anos de idade, os resultados apontaram para pouca necessidade de tratamento, com $61,2 \%$ dos dentes decíduos examinados sem nenhuma necessidade para Sobradinho e 61,7\% para município de Tavares. Dos dentes decíduos que apresentavam algum tipo de necessidade de tratamento para ambos os municípios, predominou a necessidade de remineralização. Para idade de 12 anos, não necessitavam de nenhum tipo de tratamento $60,6 \%$ e $51,7 \%$ respectivamente em Sobradinho e Tavares. A necessidade de remineralização também predominou nessa idade (Tab. 4).
Tabela 4 - Necessidades de tratamento de crianças de cinco e 12 anos de idade dos municípios de Sobradinho e de Tavares, RS, 2010

\begin{tabular}{|c|c|c|c|c|}
\hline \multirow{3}{*}{$\begin{array}{l}\text { Necessidade } \\
\text { tratamento }\end{array}$} & \multicolumn{4}{|c|}{ Município } \\
\hline & \multicolumn{2}{|c|}{ Sobradinho } & \multicolumn{2}{|c|}{ Tavares } \\
\hline & Média & $\%$ & Média & $\%$ \\
\hline \multicolumn{5}{|l|}{5 anos } \\
\hline $\begin{array}{l}\text { Restauração de } 1 \\
\text { superfície }\end{array}$ & 0,98 & 12,6 & 1,26 & 16,2 \\
\hline $\begin{array}{l}\text { Restauração de } 2 \text { ou } \\
\text { mais superfícies }\end{array}$ & 1,47 & 18,8 & 0,85 & 11,0 \\
\hline Exodontia & 0,35 & 4,5 & 0,31 & 4,0 \\
\hline Remineralização & 5,00 & 64,0 & 5,34 & 68,7 \\
\hline \multicolumn{5}{|l|}{12 anos } \\
\hline $\begin{array}{l}\text { Restauração de } 1 \\
\text { superfície }\end{array}$ & 0,31 & 3,1 & 0,39 & 3,1 \\
\hline $\begin{array}{l}\text { Restauração de } 2 \text { ou } \\
\text { mais superfície }\end{array}$ & 0,34 & 3,4 & 0,17 & 1,4 \\
\hline Tratamento pulpar & 0,04 & 0,5 & 0,12 & 1,0 \\
\hline Exodontia & 0,22 & 2,2 & 0,16 & 1,3 \\
\hline Remineralização & 9,15 & 90,8 & 11,61 & 93,1 \\
\hline
\end{tabular}

\section{Discussão}

A característica mais importante desta pesquisa é o fato de que não havia dados coletados e sistematizados para os municípios, de forma a possibilitar comparações com resultados de outros estudos realizados no Rio Grande do Sul e no Brasil. Além disso, de posse desses resultados, as autoridades sanitárias do município terão subsídios para planejar e avaliar suas políticas públicas de saúde bucal.

Alguns cuidados devem ser tomados na análise dos dados de Sobradinho por se tratar de uma amostra de conveniência, pois não se pode definir o motivo que levou à devolução das autorizações pelos pais/ responsáveis. O mais importante é que os resultados podem indicar o perfil da população estudada, auxiliando no cálculo de amostras para estudos futuros. O mesmo não aconteceu para o município de Tavares, onde foi realizado um censo com os escolares dessas idades, com um baixo percentual de perdas, assegurando a situação real da população estudada.

O processo de calibração cuidadoso e as concordâncias obtidas pelas examinadoras asseguraram a confiabilidade nos resultados obtidos. As limitações do índice CPOD, o qual avalia somente estágios mais avançados das lesões de cárie, foram minimizadas ao se incluir, na avaliação das necessidades de tratamento, a indicação de remineralização de manchas brancas de cárie, quando essas estavam presentes.

Os valores do índice ceod foram semelhantes nos dois municípios, conforme se pode observar: 2,92 para Sobradinho e 2,91 para Tavares, diferente de outro estudo realizado no nordeste do Brasil, onde o ceod foi de 4,01, o que aponta para inequi- 
dades em saúde, quando se compara com outras regiões do Brasil ${ }^{12}$. Estavam livres de cárie aos cinco anos, 32,4\% das crianças de Sobradinho e 45,7\% das de Tavares. Semelhantes aos resultados do levantamento epidemiológico sobre as condições de saúde bucal no Rio Grande do Sul (SB RS 2003), que analisando os dados da Macrorregião dos Vales, a qual pertence o município de Sobradinho, estavam livres de cárie $35,3 \%$ das crianças de cinco anos. Na Macrorregião Metropolitana, a qual pertence o município de Tavares, esse percentual foi de $46,6 \%{ }^{13}$. O $\mathrm{SiC}$ Index para cinco anos foi de 7,23 em Sobradinho e 8,30 em Tavares.

$\mathrm{Na}$ idade de 5 anos, em 2010, nenhum dos dois municípios atingiu as metas recomendadas pela OMS para o ano 2000, ficando aquém da meta da OMS para o ano de $2010^{14}$. Assim como em outros estudos ${ }^{1,12,15}$, o componente cariado do índice foi 0 mais prevalente para ambos os municípios, revelando uma deficiente cobertura na atenção odontológica para essa idade ou uma menor importância dada à dentição decídua. Os resultados do $\mathrm{SiC}$ Index ${ }^{10}$ ainda indicaram que uma pequena parcela da população pré-escolar acumula grande parte da doença, o que destaca a necessidade de investimento em estratégias de promoção da saúde, incluindo a continuidade das medidas populacionais adotadas no município e a ampliação das medidas educativas e preventivas voltadas a esse grupo populacional. Além disso, essa realidade demonstra a necessidade de ampliação do acesso ao tratamento odontológico para toda a população ${ }^{16}$.

Nas crianças de 12 anos em Sobradinho, o CPOD foi de 1,05. Embora esse índice de CPOD caracterize uma prevalência de cárie dentária muito baixa ${ }^{17}$, sabe-se que a redução do valor do índice para médias menores que um é um grande desafio para os serviços públicos de saúde. $\mathrm{O}$ fato de o componente cariado não ter sido observado nos primeiros molares permanentes aos cinco anos de idade e o componente "obturado" representar 53,3\% da composição do CPOD aos 12 anos de aponta para necessidade de ações preventivas específicas para esses dentes ${ }^{18}$, de forma a favorecer a redução das médias do índice CPOD para os próximos anos. O componente obturado apareceu como maior responsável pelo índice CPOD do município, o que pode revelar que a população tem um maior acesso aos serviços de saúde, porém indica que sejam revistas as ações coletivas de educação em saúde e prevenção da cárie dentária ${ }^{18}$.

Essas observações também são válidas para o município de Tavares, porém, em situação mais crítica por ter valores de CPOD $(2,80)$, maiores que o município de Sobradinho e apresentar 4,2\% dos primeiros molares permanentes já cariados aos cinco anos de idade.

Quando se analisa os índices de CPOD relativos aos 12 anos de idade nos dois municípios, observa-se que Sobradinho já apresenta índices menores dos que os apresentados para o Brasil ${ }^{1}$ e semelhan- te aos de outros estudos ${ }^{13,17}$. Por outro lado, Tavares encontra-se em uma situação mais desfavorável que vários locais ${ }^{2,7,15}$. Isso é reforçado ao se analisar os valores do $\mathrm{SiC}$ Index ${ }^{11}$ que em Tavares foi de 5,64, e Sobradinho, com média do CPOD próxima de um, apresenta o $\mathrm{SiC}$ Index com valor de 3,27. Ao se observar a composição do índice CPOD, nota-se que Sobradinho e Tavares têm maior proporção do componente obturado como se observa em vários outros estudos ${ }^{6,19,20}$.

No município de Tavares, apenas uma pequena parcela das crianças de 12 anos de idade estava livre de cárie $(C P O D=0)$, situação semelhante à de outro estudo realizado no Rio Grande do $\mathrm{Sul}^{20}$. Esse resultado aponta a necessidade de adequação no modelo de atenção em saúde bucal com maior investimento em necessidade de atividades preventivas e educativas.

Aos cinco anos de idade, os resultados apontaram para pouca necessidade de tratamento para os dois municípios. Dos dentes que apresentavam algum tipo de necessidade de tratamento, a maioria necessitava de remineralização de mancha branca o que diferiu de outros estudos, em que a principal necessidade de tratamento era a restauração de uma ou mais superfícies dentárias ${ }^{6,19}$.

Em relação a tratamento para crianças de 12 anos de idade, os resultados mostraram que mais da metade dos dentes permanentes examinados no município de Sobradinho e de Tavares não tinham nenhuma necessidade de tratamento. Nos dois municípios, dos que necessitavam de algum tipo de tratamento, mais de $90 \%$ precisavam de remineralização dentária semelhante a um estudo realizado em Água Santa $(\mathrm{RS})^{18}$.

Quando comparados com às necessidades de tratamento de crianças em outros estudos em que a prevalência de tratamento foi a restauração de uma ou duas superfícies ${ }^{6,13,15,17}$, os resultados encontrados apontam uma progressão mais lenta da doença. Essa situação reforça ainda mais a necessidade de ações coletivas de educação em saúde, principalmente voltadas ao controle do biofilme dental ${ }^{21}$.

\section{Conclusões}

Com relação à prevalência de cárie dentária aos 5 anos de idade, Sobradinho apresentou um ceod de 2,92 e Tavares de 2,91 com componente cariado sendo o mais prevalente nos dois municípios. Sobradinho teve $32,4 \%$ das crianças livres de cárie e em Tavares essa proporção foi de 45,7\%. Para crianças de 12 anos de idade, Sobradinho apresentou uma média de CPOD de 1,05 e Tavares de 2,80. Os componentes do índice mais prevalente para o município de Sobradinho foi o cariado que representou $44,4 \%$ e o restaurado representando 53,5\%. Tavares teve como o componente mais prevalente o restaurado com 74 , 5\%. Para a idade de 12 anos, Sobradinho teve 56,1\% de crianças livres de cárie e Tavares $16,7 \%$. 
Analisando os resultados, verificou-se que há a necessidade de diagnóstico precoce, bem como de ações preventivas coletivas e individuais, voltadas para pré-escolares, uma vez que os primeiros molares permanentes foram responsáveis por uma considerável porcentagem da composição o índice CPOD para as crianças de 12 anos de idade. Também os serviços públicos de saúde dos municípios estudados devem continuar realizando o monitoramento das condições de saúde bucal da população e desenvolver estratégias e ações de promoção de saúde que possam beneficiar a essa população.

\section{Agradecimentos}

Às Prefeituras Municipais de Sobradinho e Tavares, que aceitaram o nosso pedido.

Aos escolares e seus pais/responsáveis que concordaram em participar deste trabalho, tornando possível sua realização.

\section{Abstract}

Objective: To determine the prevalence of dental caries and treatment needs of children aged 5 and 12 years in the cities of Sobradinho and Tavares, RS, 2010. Materials and method: We conducted a cross sectional study, using the recommendations of the Word Health Organization (WHO), by two calibrated examiners (interexaminer Kappa of 0.92, 0.97 to 0.92 for examiner $A$ and examiner B). We calculated DMFT and dmft, and SiC Index for the ages and DMFT for permanent first molars. Results: We examined 115 children and 91 Sobradinho in Tavares. Were caries free at 5 years $32.4 \%$ of children Sobradinho and $45.7 \%$ of Tavares. The DMFT was 1.05 and 2.80 in Sobradinho in Tavares. The SiC Index for five years was 6.70 in Sobradinho and 7.60 in Tavares, for 12 years was 2.80 and 4.90 respectively. The need for treatment prevailing in the two cities both for 5 years to 12 years was as remineralization. Conclusions: The two cities did not meet the WHO's goal for the year 2010 to five years. For 12 years the city of Sobradinho is very close to achieving it. The treatment needs were little complex for the two municipalities. It was found that there is need for collective and individual preventive and early diagnosis aimed at pre-school since the first permanent molars were responsible for a considerable percentage of the composition of DMFT at age 12.

Keywords: Health surveys. Dental caries. Child health.

\section{Referências}

1. Brasil. Ministério da Saúde. Projeto SB Brasil 2010: Condições de saúde bucal da população brasileira 2009-2010. Resultados preliminares. Brasília; 2011.

2. Roncalli AG. Projeto SB Brasil 2010: elemento estratégico na construção de um modelo de vigilância em saúde bucal. Cad. Saúde Pública 2010; 26(3):428-9.

3. Narvai PC, Antunes JLF, Moysés SJ, Frazão P, Peres MA, Peres KG et al.Validade científica de conhecimento epidemiológico gerado com base no estudo Saúde Bucal Brasil 2003. Cad. Saúde Pública 2010; 26(4):647-70.

4. [WHO] World Health Organization. Oral health surveys: basic methods. Geneva, 3 ed.1987.

5. [WHO] World Health Organization. Oral health surveys: basic methods. Geneva, 4 ed. 1997.

6. Brasil. Ministério da Saúde. Projeto SB Brasil 2003: Condições de saúde bucal da população brasileira 2002-2003. Resultados principais. Brasília. 2003

7. Brasil. Ministério da Saúde. Sistema de Informações Ambulatoriais do Sistema Único de Saúde, 2010. Disponível em: <http://www.tabnet/Datasus.gov.br/tabdata/cadernos/ rs.htm>. Acesso em 21 ago. 2010.

8. Batista AK, Unfer B. Três olhares sobre a reorganização da atenção primária: percepção sobre o novo modelo de saúde bucal em um município do estado do Rio Grande do Sul. Saúde em Debate 2011; 35(90):417-25.

9. Eklund SA, Moller IJ, Leclercq MH. Calibration of examiners for oral health epidemiological surveys. Geneva: World Health Organization, ORH/ EPID;1993.

10. Namal N, Yüceokur AA, Can G. Significant caries index values and related factors in 5-6-year-old children in Istanbul, Turkey. East Mediterr Health J 2009; 15(1):178-84.

11. Nishi M, Stiernswärd J, Carlsson P, Bratthall D. Caries experience of some countries and areas expressed by the Significant Caries Index. Community Dent Oral Epidemiol 2002; 30(4):296-301.

12. Santos Jr VE, Alencar Filho AV, Sousa RMB, Cavalcanti F, Heimer MV, Rosenblatt A. O impacto de um programa social brasileiro sobre a saúde bucal de crianças. Rev,Fac,-Odontol Univ. Passo Fundo 2013; 18(1):61-6.

13. Rio Grande do Sul. Secretaria de Estado da Saúde. Projeto SB-RS. Condições de saúde bucal da população do Rio Grande do Sul. Porto Alegre - RS, 2001. Disponível em: <http// www.saude.rs.gov.Br/das/saúde_bucal/projeto_sb_Brasil_ gaúcho.php>. Acesso em 04 mai. 2010.

14. [OPAS/OMS] Organização Pan-Americana de Saúde/Organização Mundial da Saúde. Disponível em: <http//www.opas. org.br>. Acesso em 23 out. 2011.

15. Melo MMDC, Souza WV, Carvalho MLL, Couto GBL, Malheiros T, Paixão A. Polarização da cárie dentária em pré-escolares cadastrados no Programa Saúde da Família do Recife. Odontologia. Clín Científ 2009; 8(1):35-40.

16. Reis SCGB, Freire MCM, Higino MASP, Batista SMO, Rezende KLV, Queiroz MG. Declínio de cárie em escolares de 12 anos da rede pública de Goiânia, Goiás, Brasil, no período de 1988 a 2003. Rev Bras Epidemiol 2009; 12(1):92-8.

17. Ruiz LA, Rihs LB, Sousa MLR, Hildebrand L, Felizatti RC. Declínio da cárie dentária em escolares entre 1998 e 2004 em Leme, São Paulo, Brasil. Rev: Gaúcha Odontologia 2009; 75(2): 145-50. 
18. Moro L, Varaschini EL, Bighetti TI, Castilhos ED. Condições de saúde bucal de escolares de 12 anos de idade, município de Água Santa, Rio Grande do Sul, Brasil. Rev Fac Odontol Porto Alegre 2009; 50(2):12-17.

19. Rihs LB, Sousa MLR, Cypriano S. Abdalla NM. Desigualdades na distribuição da cárie dentária em adolescentes de Indaiatuba (SP), 2004. Ciência \& Saúde Coletiva 2010; 15(4):2173-80.

20. Rigo L, Abegg C, Bassani DG. Cárie dentária em escolares residentes em municípios do Rio Grande do Sul, Brasil, com e sem fluoretação nas águas. Rev Sul-Bras Odontol 2010; $7(1): 57-65$.

21. Brasil. Ministério da Saúde. Caderno de Atenção Básica n.17. Distrito Federal, 2006 Disponível em: <http//www.bvsms.saude.gov.br/bvs/publicacoes/abcad17.pdf $>$. Acesso em 22 out. 2011 .

Endereço para correspondência:

Denise Paiva da Rosa

Av. Duque de Caxias, 170 - apto $201 \mathrm{~K}$ - Fragata

96030-000 Pelotas-RS, Brasil

Fone: 5553 3221-0680

E-mail: nisypel@gmail.com

Recebido: 10/02/2014. Aceito: 22/05/2014. 